\title{
Les Réseaux de Soins Coordonnés: Un projet de réforme du système de soins français
}

\author{
par Robert J. Launois*
}

\section{Introduction}

Depuis plusieurs années, les dépenses de santé connaissent en France une croissance bien supérieure à celle de la richesse nationale. La consommation médicale finale, qui représentait $4,5 \%$ de la consommation des ménages en 1950 , s'élevait à $12,5 \%$ en 1985 . Simultanément, le poids des soins hospitaliers est passé de $38,3 \%$ de la consommation médicale finale en 1950 à 49,5\% en 1985, tandis que la part des soins médicaux ambulatoires régressait corrélativement de $32 \%$ à $30 \%$ et celle des achats de biens médicaux de $28,9 \%$ à $20,5 \%$.

La protection maladie est assurée à $73 \%$ par un système d'assurances sociales universel et obligatoire. Celui-ci a fonctionné jusqu'en 1979 suivant les principes traditionnels du remboursement a posteriori et du paiement à l'unité, prix de journée à l'hôpital et tarification à l'acte en médecine de ville.

Le caractère inflationniste d'un tel dispositif devenant flagrant, l'Etat français a été conduit à pratiquer une politique de rationnement budgétaire en matière hospitalière, tandis que les professions de santé cherchaient à défendre leur pouvoir d'achat par la cartellisation des prix ambulatoires (conventions). Or, les palliatifs financiers ne suffisent plus, il apparaît clair qu'une réforme de structure doit être envisagée.

Sur quels principes doit se fonder cette réforme? Quelles formes nouvelles d'organisation des soins faut-il proposer? Quels mécanismes économiques faut-il introduire pour obtenir une auto-régulation du système?

\section{Les objectifs}

\subsection{1erobjectif : maintenir les structures fondamentales de la sécurité sociale.}

Les français sont très attachés au principe de solidarité qui sous-tend notre système de sécurité sociale. Celui-ci à le mérite d'opérer une péréquation des risques au niveau national. Il n'est donc pas question de le remettre en cause fondamentalement.

* Professeur à l'Université de Rennes I, Institut La Boétie; texte présenté à l'Assemblée générale de l'Association de Genève, Berlin, 16 juin 1987. 
- Les cotisations continuent d'être fixées en proportion des salaires,

- Leur répartition entre part salariale et part patronale reste inchangée,

- Tous les mécanismes de compensation entre régimes subsistent.

Il n'y a pas de réforme du financement du système de protection sociale, ce sont les modalités de prise en charge des frais de santé qu'il convient de réviser. Il faut continuer à mutualiser les charges des assurés, tout en individualisant les recettes des producteurs en fonction des risques dont ils assument la responsabilité.

\section{2. $2^{e}$ objectif: moderniser notre appareil de distribution de soins.}

Le système de santé français ne comporte à l'heure actuelle aucune incitation à l'optimisation des choix et des moyens employés :

- Chaque médecin a un réseau de correspondants, et il entretient des relations privilégiées avec des laboratoires et des structures hospitalières privées ou publiques.

- Ces relations définissent des filières de soins que le malade emprunte en fonction des orientations dont il fait l'objet.

- Il y a bien une véritable chaîne d'intervenants qui couvrent la totalité des besoins de la population, mais cette chaîne est mal organisée.

La multiplicité des intervenants compromet la continuité des soins et pousse à la dépense.

- Les prestataires de services ne se sentent pas responsables du destin des malades dont ils ont la charge, dès lors que ceux-ci sortent de leur champ de compétences propre. Le malade est exposé à une médecine parcellisée, plus centrée sur la maladie que sur la santé de l'individu.

- Les prescripteurs ne sont pas amenés à prendre en considération les conséquences financières de leur choix, dès lors qu'ils n'en subissent pas directement les effets. Comment un généraliste pourrait-il chercher à tout prix à éviter une hospitalisation inutile, alors qu'il ne perçoit rien sur les milliers de francs dont il permet à la sécurité sociale de faire l'économie.?

La passivité des mécanismes de financement n'encourage pas à la recherche de l'efficacité. Dans la vie économique, celui qui décide est également celui qui paye et qui consomme. Dans le domaine de la santé, ces trois rôles sont dissociés. Le consommateur n'est pas le payeur, puisque c'est la sécurité sociale qui règle la facture. Il n'est pas non plus le décideur, puisqu'il agit sur prescription.

- Le consommateur n'éprouve pas le besoin de faire ses choix en fonction des coûts, puisque la plus grande partie des frais est prise en charge par l'assurance-maladie. L'assuré tend à se payer sur la bête en adoptant un comportement du type "j'ai payé, j'ai droit".

- Les prestataires de services, n'étant plus limités par les capacités financières de leur clientèle, adoptent une stratégie productiviste. La motivation à faire le meilleur usage des ressources disponibles disparaît. 
- Aucun mécanisme de prix ne vient coordonner le choix des agents composant le système. Jamais les prix médicaux n'ont été placés depuis 1945 sous un régime de complète liberté. Il sont soit cartellisés, soit administrés.

- L'encadrement des coûts en médecine de ville par le blocage des tarifs, en hospitalisation, par le budget global, est voué à l'échec. Le premier laisse le volume des actes indéterminé, le second pénalise les bons gestionnaires.

- A un moment ou à un autre, il faut bien solder les comptes et augmenter le taux des cotisations. Mais l'effet de rétroaction de ces mesures est dérisoire, puisqu'il n'y a aucun lien entre les cotisations payées par l'assuré et les dépenses dont il est à l'origine.

Pour mettre fin à ces disfonctionnements, il faut articuler financièrement toutes les opérations qui se déroulent le long de la chaîne de telle façon que les différents acteurs soient amenés à promouvoir l'intérêt de la collectivité en recherchant leur propre intérêt.

\subsection{3e objectif : promouvoir la médecine globale.}

La santé n'est pas simplement l'absence de maladie, c'est aussi la présence d'habitudes de vie saine et de conditions d'environnement favorables. Cette globalité de la santé exige une prise en charge totale de l'individu et suppose que tous les professionnels de la santé, médecins ou non, contribuent à sa défense.

L'intégration d'une attitude préventive dans le comportement professionnel est la première caractèristique de cette nouvelle médecine. "Le praticien doit cesser d'être un technicien de la maladie pour devenir un artisan de santé". Son action ne peut se limiter à la préservation et à la restauration de l'état de santé des clients qui le consultent dans le secret de son cabinet. Elle doit s'élargir à l'ensemble de la population dans laquelle sa pratique se situe, pour la mettre en garde contre des facteurs de risque dont elle ne perçoit pas a priori le danger.

L'instauration de liaisons permanentes avec des partenaires non médicaux est la seconde caractéristique de cette approche globale des problèmes de santé. Une seule personne n'est plus à même de résoudre les problèmes qui se posent au malade. Le médecin doit désormais être aidé dans sa tâche par de véritables équipes pluri-professionnelles.

L'objectif d'un tel élargissement de la pratique médicale est de limiter le recours à l'hospitalisation en modulant le degré de médicalisation des soins et d'éviter les gaspillages en concentrant les équipements. Le précédent américain des HMO montre qu'un tel objectif peut être atteint. Mais il ne suffit pas d'appeler de ses vœux un exercice coordonné de la médecine, encore faut-il mettre en place une organisation qui en permette l'émergence. La création des réseaux de soins coordonnés est la réponse institutionnelle au problème posé.

\section{L'organisation}

Un réseau peut se définir ainsi: C'est une structure de regroupement des professionnels de santé, animée par le médecin de famille, choisie par le malade, qui offre à une population d'adhérents quantitativement définie, une filière de soins complète et homogène, en contrepartie d'un prix annuel global fixé à l'avance, financé pour partie par la sécurité sociale et pour partie par l'assuré. Le risque financier - gains ou pertes constatés en fin d'année - y est partagé entre l'organisation et les médecins qui en sont membres. Au delà d'un certain seuil, il est cédé aux organismes promoteurs de l'expérimentation. 
Un contrat est passé entre les usagers et les professionnels de santé pour assurer la prise en charge complète des individus. Le client s'engage à se faire soigner exclusivement par le R.S.C. pour une durée limitée à un an. Le R.S.C. lui garantit de dispenser les soins de toute nature dont il pourrait avoir besoin. Ceci englobe aussi bien les soins curatifs que la prévention et la réadaptation.

Un tel accord est compatible, comme nous le montre l'experience HMO, avec toutes les formes de rémunération de l'activité médicale. Dans les associations de praticiens libéraux (IPA), les médecins sont rémunérés au prorata du nombre d'actes effectués, mais ceux-ci sont facturés à l'organisation et non à l'assuré. Dans les groupements multidisciplinaires prépayés (PGP, i.e. cabinets de groupe ou réseaux de cabinets de groupe), les équipes médicales perçoivent une capitation par adhérent pris en charge. Dans le modèle HMO le plus ancien (staff model), le personnel médical est salarié.

\section{Le financement}

Pour que les réseaux puissent atteindre les objectifs qui leur sont assignés, il faut mettre en place des règles de financement et de gestion qui les y incitent.

Trois mécanismes sont introduits:

- Le financement a priori per capita;

- La prise en charge forfaitaire des dépenses par la sécurité sociale;

- La concurrence entre les équipes de soins.

\subsection{Le financement a priori per capita.}

Le financement des réseaux est assuré sur la base d'un prix annuel global (PAG) par adhérent inscrit, dont le montant est fixé en toute liberté par les dirigeants de l'organisation.

Le caractère annuel du financement interdit toute révision en cours d'exercice.

- Une contrainte de coût est introduite au niveau où se forment les décisions. Ce ne sont plus, comme dans le système actuel, les dépenses constatées qui déterminent les ressources nécessaires, c'est à l'inverse la masse des ressources disponibles qui limitent les prestations offertes.

- Les procédures de gestion de clientèle sont fondamentalement modifiées. Désormais, ce ne sont plus des recettes, mais des charges qui sont créées chaque fois qu'un service est rendu au malade. L'organisation possède les caractéristiques institutionnelles qui la poussent à rechercher la plus stricte économie compatible avec l'efficacité du traitement.

- La responsabilité financière des producteurs est clairement établie, car dans le cadre d'une organisation dont le financement est déterminé ex ante, les parties impliquées sont inévitablement amenées à partager les gains et les pertes constatées en fin d'année. Toute décision thérapeutique inutile se traduit pour son auteur par une perte égale au supplément de rémunération qu'il aurait pu percevoir s'il s'était abstenu de la prendre. Ce n'est plus une administration anonyme qui rationne autoritairement les soins, c'est le médecin lui-même qui fait, au chevet du malade, les choix nécessaires pour éviter les dépenses superflues. 
Le caractère global du financement redonne aux décideurs une entière liberté de gestion.

- Les recettes perçues ne sont pas préaffectées à des prestations spécifiques ou subordonnées à la réalisation d'un sinistre particulier. Le coût de fonctionnement des réseaux est couvert sans référence à la diversité des services rendus. Il n'est plus nécessaire de quantifier l'activité médicale en la traduisant en actes type, les notions de remboursement à l'acte et à la journée disparaissent.

- La complémentarité des diverses structures de prise en charge peut être exploitée au mieux dans le cadre d'une gestion financière unifiée. Les dirigeants du RSC doivent faire des choix explicites entre les diverses formes de soins, soins ambulatoires, alternatives à l'hospitalisation, hospitalisation. La rationnalisation du système se fait par grandes masses, et non par un contrôle pointilliste sur les actes.

\subsection{La prise en charge forfaitaire des dépenses par la sécurité sociale.}

Les caisses d'assurance-maladie participent au financement du prix de la prise en charge par le versement d'un forfait annuel de santé (FAS), dont le montant est identique quel que soit le réseau choisi, mais dont la valeur varie en fonction de l'âge et du sexe de la personne prise en charge.

Pour fixer le montant de sa participation, la sécurité sociale doit :

- En premier lieu, délimiter de manière objective les dépenses dont elles se porte garante. A cette fin son département statistique calcule chaque année le coût actuariel national des soins, ou CANS, par grandes catégories d'assurés. Il correspond, dans les statistiques de la CNAM, à la dépense moyenne reconnue par personne protégée. En sont donc exclus les dépassements d'honoraires et l'auto-médication, qui ne font l'objet d'aucun remboursement dans le cadre de la législation actuelle.

- En second lieu, elle doit fixer le pourcentage de ses dépenses qu'elle prendra en charge en versant un forfait annuel de santé (FAS) pour tout adhérent inscrit dans un réseau. La contribution des caisses ne peut couvrir l'intégralité du coût actuariel. En effet, les ménages financent encore une partie non négligeable de la dépense reconnue : $11,6 \%$ pour les enfants de sexe masculin de moins de neuf ans, 3,3\% pour les hommes de plus de 80 ans, en 1982. Il convient donc de moduler le financement des caisses selon les classes d'âge et de sexe pour maintenir les charges de l'assurance-maladie à leur niveau actuel. L'inscription d'un homme âgé de plus de 80 ans aurait entraîné en cette même année 1982 le versement de $12987 \mathrm{~F}$. Par contre, la valeur du forfait acquitté pour un enfant de moins de 12 ans aurait été seulement de $2324 \mathrm{~F}$.

L'intérêt d'une telle formule de financement est double. La valeur des forfaits augmente avec l'importance des besoins de la population prise en charge. Dès lors, les recettes des réseaux sont proportionnelles à leur coût et la tentation de l'écrémage des bons risques peut être évitée. Les forfaits sont acquis de manière définitive par les réseaux auxquels ils ont été versés. Ceux-ci en conservent la disposition en l'absence de tout recours aux soins des personnes protégées. Ainsi se trouve opérée une péréquation entre les adhérents qui permet de financer les traitements les plus coûteux. 
Pour s'inscrire dans un réseau, l'adhérent potentiel verse la différence entre le prix annuel global de la prise en charge et le montant du forfait versé. Sa participation est payable d'avance et une fois pour toutes. Il y a simple substitution d'un ticket modérateur ex ante à un ticket modérateur ex post. Puisque le réseau fixe son prix en toute liberté, la valeur de celui-ci peut être supérieure au coût actuariel reconnu par la CNAM. Auquel cas, à un ticket modérateur de fait s'ajoute un ticket modérateur de droit. Un principe d'économicité est introduit dans le choix des usagers. Ceux-ci sont incités à faire pression sur les producteurs de soins pour obtenir une réduction de leur participation. Mais en même temps, toutes les aspirations individuelles peuvent s'exprimer dans leur diversité.

Une distinction claire est donc introduite entre le tarif de prise en charge, qui dépend du bon fonctionnement des équipes de soins, et les allocations versées par les caisses pour en assurer le financement, dont le montant est calculé en fonction des besoins de la population et stratifié par âge et par sexe. L'assuré, qui ne verse que la différence entre ces deux montants, est financièrement responsabilisé. Mais la solidarité est pour l'essentiel sauvegardée:

- Solidarité entre les chanceux et les malchanceux à l'intérieur d'une classe de risques, puisque la sécurité sociale verse un forfait annuel dont le montant est indépendant de la vulnérabilité personnelle des individus qui en relèvent.

- Solidarité entre les bons et les mauvais risques à l'intérieur d'un réseau, puisque chacun paie la même contribution forfaitaire personnelle.

- Solidarité entre les riches et les pauvres au niveau collectif, puisqu'une partie des cotisations prélevées en fonction du niveau de salaire est utilisée pour financer les forfaits annuels de santé des plus forts consommateurs, qui sont des personnes âgées en général fort démunies.

\subsection{La mise en concurrence des équipes de soins.}

Le paiement par l'adhérent de sa contribution personnelle apporte au réseau le forfait correspondant de la sécurité sociale. L'intervention des payeurs n'existe que du fait des assurés, puisque les versements ne sont opérés qu'en fonction de leur choix. Il n'y a pas de financement direct des prestataires de services, le spectre de la sectorisation et de la médecine de caisses est écartée. Le libre choix de l'organisme prestataire introduit une saine émulation entre les équipes de soins, une concurrence bénéfique s'instaure à un triple niveau :

- Entre les RSC vis-à-vis de leurs adhérents potentiels : les ressources du réseau dépendent du nombre de ses adhérents, pour les attirer, il doit minimiser le montant de leur participation personnelle. Pour les conserver, il ne doit pas les décevoir sur le plan de la qualité. Celui qui a le meilleur rapport qualité des soins/participation personnelle des assurés attire la clientèle. En un mot, l'adhérent paie avec l'impression d'en avoir ou de ne pas en avoir pour son argent.

- Entre les professionnels de santé vis-à-vis des RSC : si un producteur est trop cher, le réseau devra augmenter ses prix, et deviendra non compétitif. Seuls les producteurs les moins onéreux seront agréés. 
- Entre l'ensemble des RSC et les autres formes de distribution de soins : c'est à l'usager de faire le choix du type de médecine qu'il veut et d'en payer le prix. C'est au praticien de faire le choix de la forme d'exercice qu'il entend pratiquer et d'en prendre les risques. Rien n'est imposé, à défaut de résultats probants, les expériences proposées dispraîtront d'elles-mêmes au bout de quelques années.

Ce qui fait l'originalité des réseaux par rapport aux $\mathrm{HMO}$, ce ne sont ni les objectifs qui leur sont assignés, ni les moyens proposés pour les réaliser. Les HMO mettent les mêmes techniques (filières de soins intégrées, médecine pré-payée, intéressement aux résultats) au service des mêmes fins (promotion de la médecine globale, rationnalisation du système de santé). Mais là s'arrêtent les ressemblances. Dans le système réseaux, c'est la sécurité sociale qui joue le rôle de tiers garant, alors que dans les HMO, c'est soit l'État, soit les entreprises qui supportent le coût des prises en charge. Lorsque l'État fédéral finance l'inscription des personnes âgées de plus de 65 ans dans une HMO, il donne un exemple éclatant de solidarité nationale, mais la liberté des prix disparaît : la participation de Medicare est en effet plafonnée à $95 \%$ du montant de la dépense moyenne remboursée par personne protégée dans le système de couverture et de soins traditionnel. Lorsque ce sont les entreprises qui participent au financement de la protection sociale de leur salariés, la liberté des prix est restaurée puisque les HMO fixent librement le montant des primes appelées, mais la solidarité se réduit, car l'importance des contributions patronales varie au gré des conventions collectives en fonction de la puissance respective des partenaires sociaux.

Le modèle RSC fusionne les deux dispositifs et offre une solution originale. Il s'inspire du premier pour proposer un versement par les caisses d'un forfait nivelé par tranches d'âge, le FAS. Du second, il retient l'idée que le tarif de prise en charge peut-être dissocié des allocations versées pour en assurer le financement. La règle des $3 \mathrm{U}$ : unité, universalité, uniformité, sur laquelle repose la sécurité sociale française, est respectée dans le maintien de la liberté des prix. La concurrence entre les équipes de soins acquiert un contenu : le ticket modérateur a priori. La stratégie de marché proposée par Alain Enthoven trouve dès lors naturellement sa place dans un système de financement socialisé.

A un moment où le rationnement de la santé par la contrainte publique est de moins en moins bien supporté par les usagers et les professionnels, la création d'un quasi-marché avec réseaux de soins coordonnés, financés à priori dans le maintien des structures fondamentales de la sécurité sociale, apparaît comme un moyen de donner une plus grande efficacité financière et humaine au système sanitaire français.

\section{BIBLIOGRAPHIE}

GIRAUD, P. et R.J. LAUNOIS, Les réseaux de soins, Médecine de demain, Economica 1985.

R.J. LAUNOIS, B. MAJONONI D'INTIGNANO, V. RODWIN, J.C. STEPHAN, "Les réseaux de soins coordonnés : proposition pour une réforme profonde du système de santé". Revue française des Affaires Sociales, № 1, 1985.

R.J. LAUNOIS, "Les réseaux de soins coordonnés", in Systèmes de santé, HMO, RSC, et comparaions internationales. Institut La Boétie, 1985.

R.J. LAUNOIS, D. TRUCHET "Vers une implantation des réseaux de soins coordonnés" in Journal d'économie médicale vol 4 no 3-4, mai-août 1985, no spécial "Les R.S.C.: Une organisation du système de santé". 